\title{
Long Term Stability of Miniature Fixed-Point Cells Used in Self-Calibrating Thermometers
}

\author{
G. Krapf, M. Schalles, H. Mammen, F. Hilbrunner, S. Augustin, G. Blumroeder, T. Froehlich \\ IImenau Technical University, Institute of Process Measurement and Sensor Technology \\ Gustav-Kirchhoff-Strasse 1, 98693 IImenau
}

\begin{abstract}
In many temperature critical and security-relevant industrial processes the drift of the characteristics of the installed temperature sensors has a dominant effect on the resulting measurement uncertainty. Also many applications can be found in medical technology, pharmaceutics, biotechnology and food industry where an exact and traceable knowledge of the process temperature is indispensable. For this reason a frequent calibration of the used sensors becomes necessary. During this calibration process the temperature sensors usually have to be taken out of the process to be measured. In doing so, they can only be calibrated without their measurement chain. This often causes additional non-negligible contributions to the calibration uncertainty. Here and in other cases a metrological traceable in-situ calibration can improve the achievable measurement and calibration uncertainty.

Against this background, self-calibrating industrial thermometers containing miniaturised fixed-point cells were developed, improved and tested under operating conditions during the last years. Within this research a large number of miniature fixed-point cells were filled with zinc of different purities. These cells were aged under tough operating conditions partly in an oxidising atmosphere. The whole ageing process lasted more than a year. Within this timespan the zinc phase transition temperatures were monitored periodically and analysed. For this the cell temperatures were measured by mineral insulated resistance thermometers while the cells were heated up and cooled down at different heating and cooling rates. The thermometers were continuously calibrated at a traceable standard zinc fixed-point.

This article contains the collected data as well as their analysis of the ageing. It also assesses the time-dependent calibration uncertainty of self-calibrating thermometers on the basis of miniature fixedpoint cells.
\end{abstract}

\section{Intention}

Temperature sensors and their measurement chains always underlie certain drift and hysteresis effects specific to the application. Especially at higher process temperatures this becomes a key influence on the obtainable measurement uncertainty. To meet the uncertainty demands of the application it is often necessary to calibrate the thermometers periodically or even to replace them. Additionally the thermometer calibration itself can cause subsequent components of uncertainty in many cases. One example is the thermal connection between the thermometers and the calibration furnace that can be different from the real process. Furthermore the lateral temperature profile in the calibrator as well as the heat dissipation often differs from the practical application. Also the entire measurement chain can rarely be calibrated in complex plants. All of these uncertainty components could be estimated and corrected by an in-situ calibration of the thermometer directly in the application by means of thermometers with integrated miniaturised fixed-point cells (MFPC) [1].

These MFPC's consist of a crucible made of aluminium oxide ceramics, which is filled with a small amount of a highly pure metal (for example $650 \mathrm{mg}$ zinc) or a binary metal alloy (see fig. 1). A reentrant tube made of aluminium oxide or aluminium nitride ceramics is arranged inside this fixed-point cell. In this tube a thermocouple or resistance thermometer is positioned. This temperature sensor and its connecting wires are guided by a ceramic capillary, which guarantees an electrical insulation and a reproducible positioning of the sensor in the reentrant tube. The cell itself is enclosed by an electrical heating element, which is surrounded by a ceramic insulator. The entire arrangement is assembled in an industrial casing tube with an outside diameter of $6 \mathrm{~mm}[2]$. 
With the help of the integrated heating element the temperature of the fixed-point cell can be raised above the process temperature until the melting point of the fixed-point substance is exceeded. During the melting a plateau in the measured time-dependant temperature curve develops (fig. 2). Its inflection point temperature can be used as a calibration value for the integrated sensor element [4].

Analogous to the melting, the solidification of the fixed-point material can be used for the calibration of the internal sensor. However the undercooling of the fixed-point material can lead to systematic measurement errors. Therefore freezing points are only conditionally used for the calibration by means of self-calibrating thermometers.

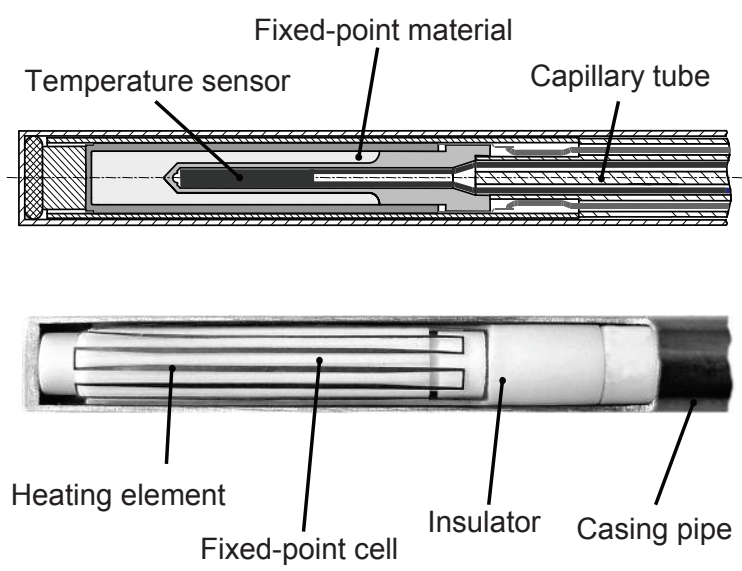

Fig. 1 Sectional view of a thermometer with an integrated fixed-point cell (MFPC)

To achieve minimal errors in subsequent measurements it is necessary to have the melting point of the fixed-point substance closely above the application temperature. Since this cannot be realised only by using pure elements as fixed-point substances, also binary eutectic alloys are utilised in self-calibrating thermometers [3].

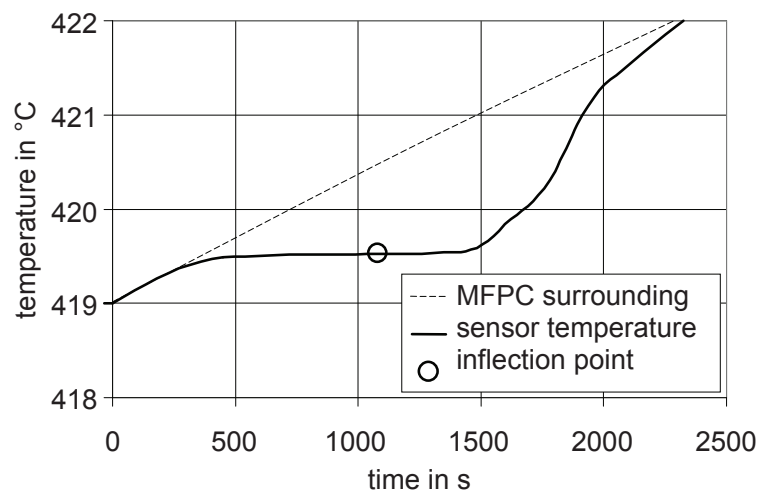

Fig. 2 Melting plateau of a self-calibrating thermometer with zinc filled MFPC

After the industrial testing of self-calibrating thermometers within the last years further important questions arose. Hence the best attainable calibration uncertainty, the mechanical-thermal long-term stability of the MFPC, the ageing as well as the reactions of crucible material and the fixed-point substance had to be researched more precisely. For these investigations zinc was selected as fixed-point material, since its solidification temperature is one of the defining fixed-points of the International Temperature Scale, ITS-90. Using a standard zinc fixed-point as reference, a direct and reliable transfer of the measured MFPC temperatures to the international thermometric scale can be carried out. 


\section{Measurement set-up}

For the investigations 33 miniature fixed-point cells made of highly pure aluminium oxide ceramics were filled with zinc of $3 \mathrm{~N}$ (zinc content $99.9 \%)$ to $6 \mathrm{~N}(99.9999 \%$ zinc) purity. These cells were arranged in a ceramic holder, which was positioned in an upright tubular furnace (fig. 4a, c).
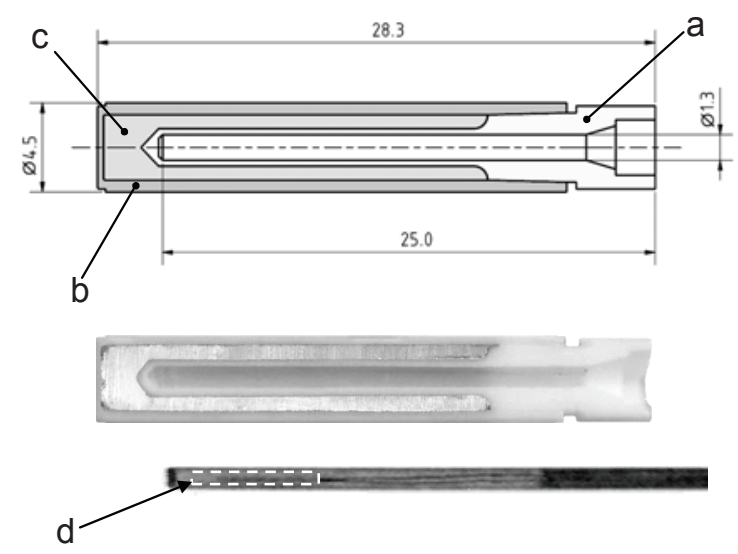

Fig. 3 Sectional view of a miniaturised fixed-point cell and $X$-ray image of used PRTs:

$a$ - reentrant tube, $b$ - exterior crucible, $c$ - fixed-point material, $d$ - sensor element

The phase transition temperatures in the fixed-point cells were measured by means of Pt100 mineral insulated resistance thermometers (PRT). They had a diameter of $1 \mathrm{~mm}$ and a length of $500 \mathrm{~mm}$ (fig. 4d). The reproducible positioning of these resistance thermometers was ensured by a ceramic guidance (fig. 4b). Furthermore springy suspensions provided constant pressure strength between the PRTs and the fixed-point cells. The measurement of the sensor resistance was carried out in relation to a $250 \mathrm{ohm}$ resistance standard by using an adapted precision current source, a high precision low-thermovoltage multiplexer and an Agilent 3458A multimeter. Self-heating effects as well as parasitic thermoelectric voltages were taken into account.

On the basis of this whole set-up it was possible to melt or freeze the fixed-point material in all cells simultaneously at heating rates from $0.01 \mathrm{mK}$ to $0.1 \mathrm{mK}$, while the corresponding phase transition temperatures were measured synchronously. In this way about 24 phase transitions were performed at every of the seven measuring cycles.
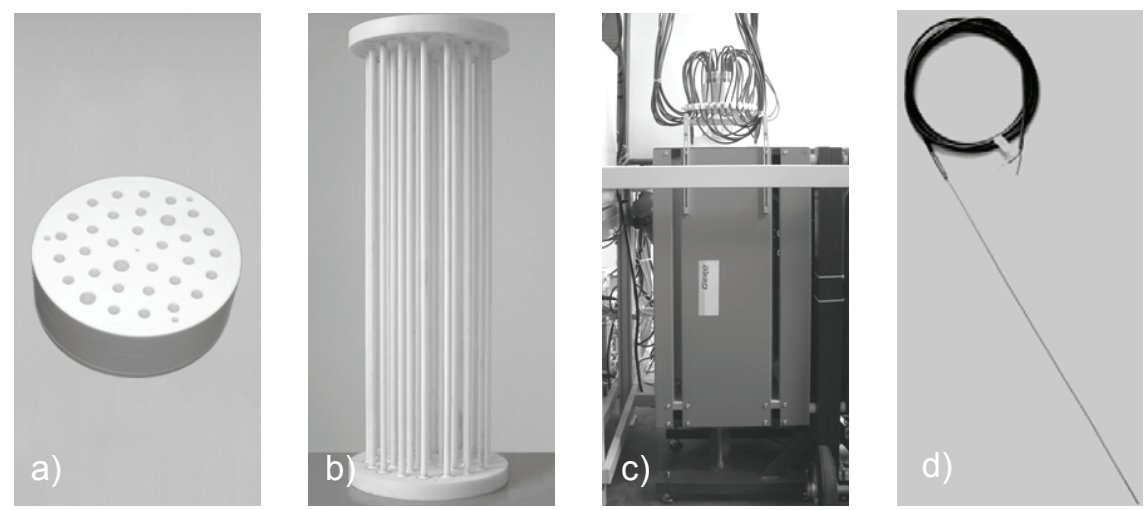

Fig. 4 Test set-up: a - MFPC holder, $\mathrm{b}$ - thermometer guidance,

$c-$ heating furnace with inserted thermometers, $d-$ Pt100 resistance thermometer

After every measuring cycle the fixed-point cells were aged in the same furnace in inert gas or oxidizing atmosphere at approximately $520^{\circ} \mathrm{C}$. For that purpose the PRTs were removed and partly replaced by wires made of similar high temperature materials which acted as a source of impurities. While the cells were aged, the PRT's were calibrated using an ice point and a certified standard zinc fixed-point. Subsequently, the freshly calibrated sensors were inserted into the MFPCs again and a new measuring cycle was started. In this manner it was possible to pass six ageing steps over a period of 18 month and to monitor the behaviour or rather the drift of the phase transition temperatures. Parallel to this the cells 
were regularly taken out of the cell holder and were weighed in by using a Sartorius Genius analytical balance. Additionally the composition of the used zinc was analysed before and after the ageing process by Glow Discharge Mass Spectrometry (GD-MS) as well as Inductive Coupled Plasma Mass Spectrometry (ICP-MS).

\section{Measurement results}

At the beginning of the investigations the examined miniature fixed-point cells were filled with zinc of different purities. Nevertheless the $4 \mathrm{~N}-, 5 \mathrm{~N}$ - and $6 \mathrm{~N}$-zinc filled cells showed almost the same behaviour during the thermal ageing. Also the fixed-point crucibles that were filled with comparative impure $3 \mathrm{~N}$-zinc behaved similarly - apart from the systematic deviations due to contained impurities. Therefore the results are discussed in principle on the example of a selected $6 \mathrm{~N}$-zinc filled cell.

The inflection points of the melting plateaus and the maxima of the freezing plateaus are shown exemplarily in figure 6 and figure 7 . It can be seen that there is no significant drift during the ageing process regarding the achievable Pt100 measurement uncertainty of $\pm 23 \mathrm{mK}(k=2)$. However the freezing temperatures show a systematic deviation from the expected solidification temperature of zinc, which is defined as $T_{90, Z n}=419,527^{\circ} \mathrm{C}$. This behaviour is typical for MFPCs. It is caused by the undercooling of the fixed-point material which can amount some ten millikelvin. In addition, the relatively small released latent energy of solidification due to the small amount of phase transition material cannot heat up the whole thermal environment of the cell.

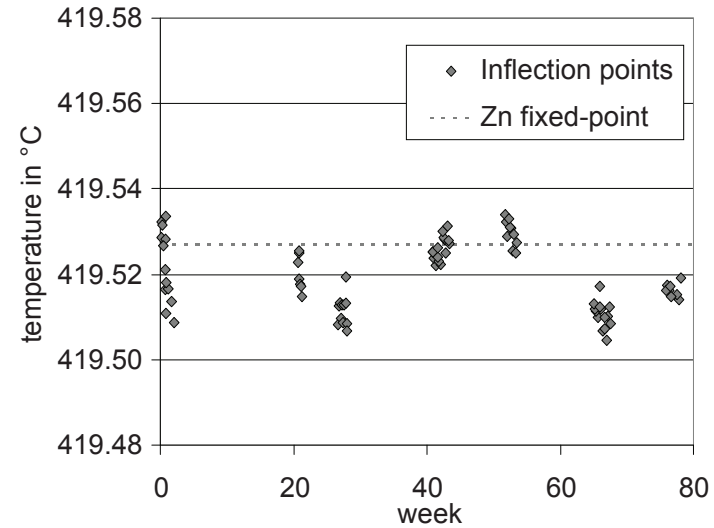

Fig. 6 Inflection points of a 6N-zinc filled MFPC's melting plateaus $-T_{\text {mean, melt }}=419.519^{\circ} \mathrm{C}$, experimental standard deviation $s_{\text {melt }}=8.4 \mathrm{mK}$

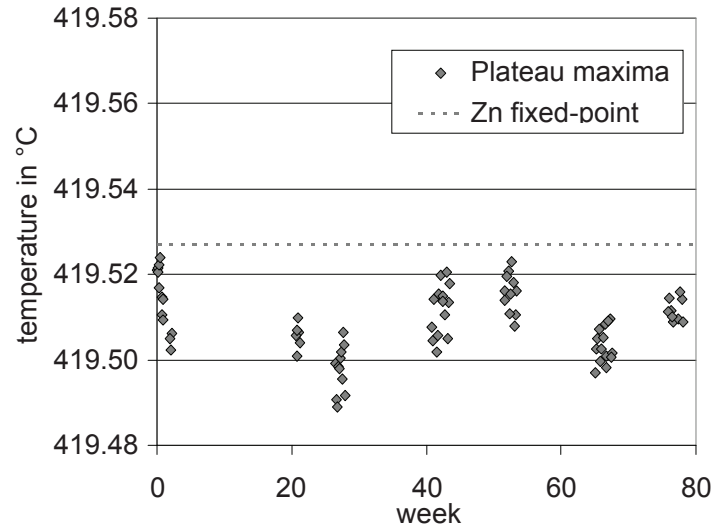

Fig. 7 Maxima of a $6 \mathrm{~N}$ zinc-filled MFPC's freezing plateaus $-T_{\text {mean,freeze }}=419.509{ }^{\circ} \mathrm{C}$, experimental standard deviation $S_{\text {freeze }}=7.9 \mathrm{mK}$

The deviations among the seven measuring cycles are primarily caused by the measurement and calibration uncertainty of the used thermometers. In addition, the varying thermal contact between the fixed-point material and the temperature sensor has also a determining influence. The reason for this can be seen in figure 5 .

a)

b)

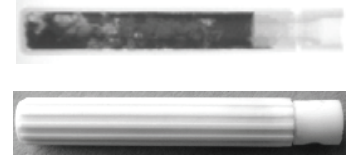

c)

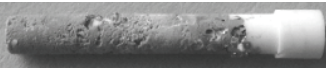

Fig. 5 MFPC after ageing process: a - X-ray image, $b$ - cell picture, $c$ - cell without outer crucible

After the filling of the cells, the zinc occupies a compact volume on radiographs. During operation a multiplicity of smaller gas inclusions developed, although the cells were aged in the same vertical installation position (fig. 5a). They can clearly be seen after disassembling the cells (fig. 5c). These inclusions significantly influence the heat transfer through the reentrant tube and lead to irreproducible measurement errors. 
Furthermore a continuous increase of the cell mass was observed at cells which were aged under oxidising atmosphere during the ageing experiment (fig. 8). This is obviously caused by the oxidation of the fixed-point material in unsealed cells. If this increase in mass would be caused solely by oxidation, one can estimate a remaining amount of $87 \%$ elemental zinc in the cell after ageing. This reduction by $13 \%$ had no remarkable influence on the measured phase transition temperatures, but the operation time of the cells is limited in our case. Which reduction would occur under industrial conditions cannot be estimated at this point and needs to be investigated in a separate test.

The mass spectrometry analyses generally showed no significant increase of impurities in zinc. Only an increased content of aluminium was detected in the probes. However the analysis methods do not provide information about the chemical bonding. Very likely the detected aluminium results from aluminium oxide, broken from the crucible surface. This can be seen in figure 9. Corresponding concentrations of elemental aluminium in zinc would lead to a depression of the phase transition temperature by some ten millikelvin, what was not observed in the measurements.

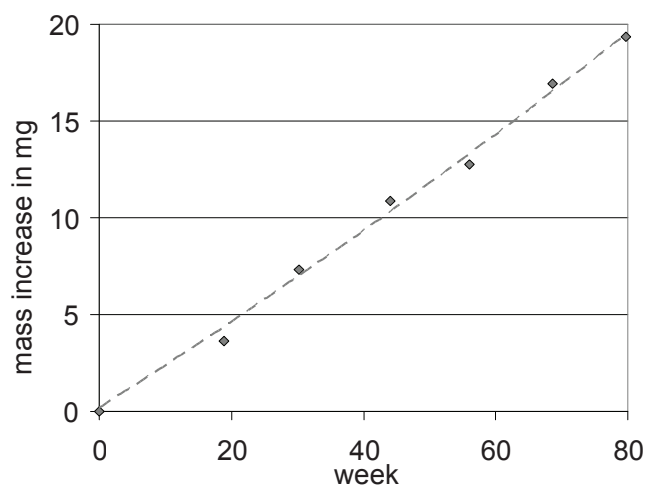

Fig. 8 Mass increase during ageing of a MFPC filled with $640.6 \mathrm{mg}$ of $6 \mathrm{~N}$-zinc

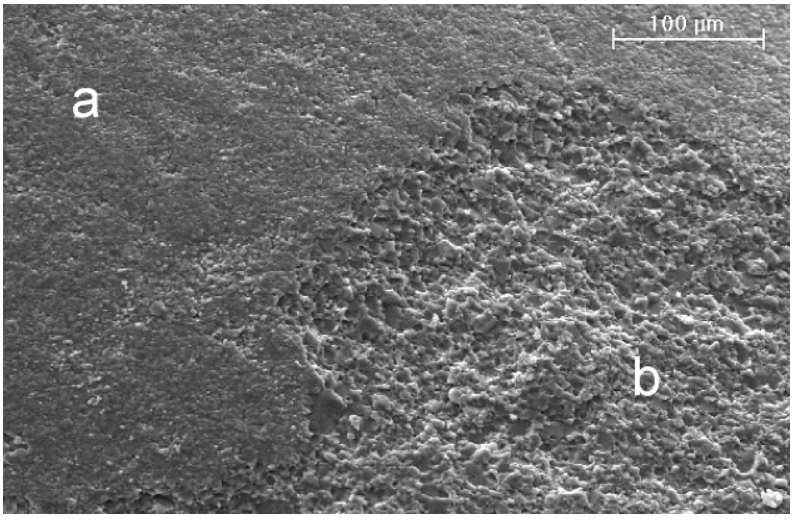

Fig. 9 SEM micrograph of the reentrant tube surface: $\mathrm{a}$ - intact $\mathrm{Al}_{2} \mathrm{O}_{3}$-ceramic surface, $\mathrm{b}$ - disruption due to adhesion of zinc in conjunction with mechanic-thermal strain

The $\mathrm{Al}_{2} \mathrm{O}_{3}$ ceramic surface is partly wetted by zinc in the molten state. This leads to a mechanical link between the ceramic surface and the zinc during solidification. Upon further cooling the zinc contracts more than the ceramics. This can lead to too strong mechanical stress and to the disruption of the ceramic surface at the end. However it had no notable influence on the measured phase transition temperatures during ageing, as already mentioned.

\section{Conclusions}

The investigations of more than 30 miniature fixed-point cells showed a good thermometric and thermo-mechanical long-term stability of ceramic cells filled with high purity zinc. Despite an operation under extreme conditions, harder than expected in typical applications, no significant drift was detected. The inflection point temperature of the melting plateau and the maximum temperature of the freezing plateau were stable with respect to the measuring uncertainty. They therefore can be used for calibration of self-calibrating thermometers. In our investigations the influence of fixed-point material oxidation and its chemical reaction with the crucible as well as accumulations of impurities had a negligible influence on the long-term stability of the phase transition temperature and the crucible material. Furthermore it was found out that the characteristics of the used mineral insulated resistance thermometers were stable and low drifting at $420^{\circ} \mathrm{C}$ as well.

The investigations showed that the concept of self-calibrating thermocouples and resistance thermometers with integrated aluminium oxide fixed-point cells excellently meets current and future industrial requirements. Moreover, the fixed-point cells can be used as economy-priced, direct traceable alternative to standard fixed-point cells in calibration laboratories, science and research. 


\section{Acknowledgements}

This research work was done within a research programme funded by the "Deutsche Forschungsgemeinschaft" DFG. The authors gratefully acknowledge the financial and administrative support.

\section{References}

[1] BogUhn, D. ET AL.: Miniature Fixed-Point Thermocouples Applicable for Industrial Purposes. In: Proceedings Volume 1, "8th International Symposium on Temperature and Thermal Measurements in Industry and Science - TEMPMEKO", Berlin, 2001. VDE-Verlag Berlin, 2002, S. 3-8

[2] Augustin, S.; Boguhn, D.: Phase Transformation Temperatures of Binary Alloys in Miniature FixedPoint Cells. In: "8th International Symposium on Temperature and Thermal Measurements in Industry and Science - TEMPMEKO", Berlin, 2001, Proceedings Vol. 2. VDE-Verlag Berlin, 2002, S. 699-704

[3] Bernhard, F.; Augustin, S.; Mammen, H.: Application of self-calibrating thermometers with miniature fixed-point cells in a temperature range from $300^{\circ} \mathrm{C}$ to $650^{\circ} \mathrm{C}$. In: "9th International Symposium on Temperature and Thermal Measurements in Industry and Science - TEMPMEKO", Dubrovnik, 2004, Proceedings Vol. 2. Zagreb : Laboratory for Process Measurement, Faculty of Mechanical Engineering and Naval Architecture, 2004, S. 1285-1290

[4] KRAPF, G.; Schalles, M.; Froehlich, T.: Estimation of Fixed-Point Temperatures - A Practical Approach. In: Measurement - Journal of the International Measurement Confederation 44 (2011), Nr. 2. Amsterdam : Elsevier Ltd., 2010, ISSN 0263-2241, S. 385-390 